\title{
MOF-Based Catalysts for Selective Hydrogenolysis of Carbon-Oxygen Ether Bonds
}

\author{
Vitalie Stavila, ${ }^{[\mathrm{a}]} *$ Ramakrishnan Parthasarathi, ${ }^{[\mathrm{a}],[\mathrm{b}]}$ Ryan W. Davis, ${ }^{[\mathrm{a}]}$ Farid El Gabaly, ${ }^{[\mathrm{a}]}$ \\ Kenneth L. Sale, ${ }^{[\mathrm{a}],[\mathrm{b}]}$ Blake A. Simmons, ${ }^{[\mathrm{b}]}$ Seema Singh, ${ }^{[\mathrm{b}]}$ Mark D. Allendorf ${ }^{[\mathrm{a}] *}$ \\ [a] Sandia National Laboratories, 7011 East Avenue, Livermore, CA 94550, * E-mail: \\ vnstavi@sandia.gov; mdallen@sandia.gov \\ [b] Joint BioEnergy Institute, 5885 Hollis Street, Emeryville, CA 94608;
}

\section{Table of Contents:}

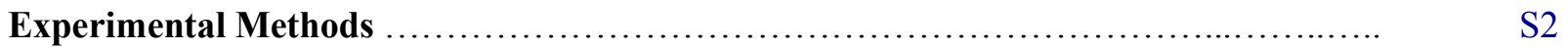

Figure S1. Gas chromatograms of the hydrogenolysis products $\ldots \ldots \ldots \ldots \ldots \ldots \ldots \ldots \ldots . . \ldots \ldots$

Figure S2. ${ }^{1} \mathrm{H}$ NMR data of the hydrogenolysis products.............................

Figure S3. Conversion efficiencies in the presence of different MOF catalysts .... S6

Table S1. Ni@IRMOF-74(I) catalytic cycling and tests in the absence of catalyst...... S6

Figure S4. SEM and EDS data for as-synthesized Ni@IRMOF-74(I) .................... $\quad$ S7

Figure S5(A,B). XPS spectra of Ti@IRMOF-74(I) and Ni@IRMOF-74(I) S8

Figure S5(C). Expanded Ti and Ni 2p regions of XPS spectra $\quad$ S9

Figure S6. Residual Gas Analysis measurements for reaction volatiles $\quad$ S9

Computational Methods .................................................. $\quad$ S10

Figure S7. Structure of IRMOF-74(I) cluster used in calculations ...................... S12

Figure S8. Structure and the pore size of IRMOF-74(I) and IRMOF-74(II)................ S13

Table S2. Optimized geometries and calculated gas-phase bond dissociation energies of ether S14 linkages in aromatic ether compounds

Figure S9. Optimized geometries of substrate-MOF cluster units ..................

Table S3. Calculated desorption enthalpies $(\mathrm{kJ} / \mathrm{mol})$ at $393 \mathrm{~K}$ of the reactants $\left(\mathrm{H}_{2}+\right.$ model $\quad \mathrm{S} 15$

compound) and products (hydrocarbon + phenol) on the IRMOF-74 cluster model

Figure S10. Molecular electrostatic potential map (MESP) of aromatic ether compounds $\quad$ S16

Table S4. Charge Transfer $(\Delta \mathrm{N})$ between aromatic ether compounds and MOF ............... S17

References ............................................................... S18 


\section{Experimental Methods}

Synthesis. All air-sensitive manipulations were performed using standard glove-box and Schlenk line techniques under argon. All chemicals and solvents were obtained from commercial sources. IRMOF-74(II)-Mg was isolated and activated following the published literature procedure using the reaction between $\mathrm{Mg}\left(\mathrm{NO}_{3}\right)_{2} \cdot 6 \mathrm{H} 2 \mathrm{O}$ and 3,3'-dihydroxy-[1,1'biphenyl]-4,4'-dicarboxylic acid in DMF. ${ }^{1}$ IRMOF-74(I)-Mg was synthesized from a solvothermal reaction of 2,5-dihydroxyterephthalic acid (98\%, from Aldrich) with magnesium nitrate $(99 \%$, Aldrich) in a mixture of $\mathrm{N}, \mathrm{N}$-dimethylformamide (DMF) $(99 \%$, Acros), absolute ethanol (99.5\%, Aldrich), and deionized water using a slightly modified literature protocol. ${ }^{2}$ $\mathrm{Mg}\left(\mathrm{NO}_{3}\right)_{2} \cdot 6 \mathrm{H}_{2} \mathrm{O}(0.729 \mathrm{~g}, 2.84 \mathrm{mmol})$ and 2,5-dihydroxyterephthalic acid $(0.198 \mathrm{~g}, 1.0 \mathrm{mmol})$ were dissolved under sonication in a 20:1:1 (v/v/v) mixture of DMF $(80 \mathrm{~mL})$, ethanol $(4 \mathrm{~mL})$, and water $(4 \mathrm{~mL})$. The homogeneous solution was then transferred to a $150 \mathrm{~mL}$ Teflon-lined stainless-steel autoclave. The autoclave was capped tightly and heated to $125{ }^{\circ} \mathrm{C}$ in an oven. After 24 hours the autoclave was removed from the oven and a yellow microcrystalline material was recovered and washed with $10 \mathrm{~mL}$ DMF. The product was then soaked in $20 \mathrm{~mL}$ DMF and heated to $80^{\circ} \mathrm{C}$ for 4 hours. The solvent was carefully decanted from the product and replaced with $20 \mathrm{~mL}$ methanol and stirred for 2 hours. Fresh methanol was used for solvent exchange for four more times. The yellow precipitate was isolated by filtration and washed thoroughly with methanol. The MOF was activated under a dynamic vacuum at $195{ }^{\circ} \mathrm{C}$ for 16 hours, yielding a yellow crystalline material.

MOF stability in various solvents. A mixture of activated IRMOF-74(I) powder (500 mg) in $20 \mathrm{~g}$ of the corresponding solvent (p-xylene, the ionic liquid 3-buthyl-1-methyl-imidazolium acetate, or water) was placed in a glass flask equipped with a magnetic stirrer. The suspensions were stirred for 16 hours at $300 \mathrm{rpm}$ at room temperature, then the solvent was removed and the MOF powders were washed with methanol and activated in vacuum. Weighting the recovered powders revealed that $>99 \%$ of IRMOF-74(I) was recovered from the p-xylene and 3-buthyl-1methyl-imidazolium acetate mixtures, while in the case of IRMOF-74(I) in water only $79 \%$ or the powder was recovered, suggesting significant MOF dissolution. All recovered powders display XRD patterns essentially identical with the powder XRD of starting IRMOF-74(I) presented in Figure 1.

Guest species infiltration. $\mathrm{TiCl}_{4}$ and $\mathrm{Cp}_{2} \mathrm{Ni}$ were loaded into the pores of IRMOF-74(I) and IRMOF-74(II) using vapor infiltration. First, the as-activated MOF was infiltrated with $\mathrm{TiCl}_{4}$ or $\mathrm{Cp}_{2} \mathrm{Ni}$ vapors at $90{ }^{\circ} \mathrm{C}$ overnight. Next, the infiltrated IRMOF-74 material was loaded and sealed in a stainless-steel autoclave. The sealed autoclave was evacuated and filled with gaseous hydrogen at 1.0 MPa pressure. Then, the autoclave was heated to $95{ }^{\circ} \mathrm{C}$ and kept at that temperature for 2 hours. The catalyst samples obtained after impregnation with $\mathrm{Ni}$ and $\mathrm{Ti}$ species are referred hereafter as Ni@IRMOF-74(X) and Ti@IRMOF-74(X), where X = I or II. Nitrogen BET analysis indicates that the surface area of IRMOF-74(I) and IRMOF-74(II) is reduced from $1627 \mathrm{~m}^{2} / \mathrm{g}$ to 431 and $459 \mathrm{~m}^{2} / \mathrm{g}$ upon infiltration with $\mathrm{Ti}$ and $\mathrm{Ni}$ species, respectively. In the case of IRMOF-74(II) the surface area decreases from $1736 \mathrm{~m}^{2} / \mathrm{g}$ for the activated IRMOF-74(II) to 672 and $591 \mathrm{~m}^{2} / \mathrm{g}$ for Ti@IRMOF-74(II) and Ni@IRMOF-74(II), 
respectively.

Catalytic tests. The catalytic hydrogenolysis reactions were performed in a stainless steel reactor equipped with a hydrogen feed. In a typical procedure, $45 \mathrm{mg}$ catalysts powder is loaded inside the reactor. A solution of the corresponding substrate (PPE, BPE or DPE) in $5.0 \mathrm{~mL}$ xylene is then added to the reactor. The reactor is pressurized with gaseous hydrogen, then sealed. The reactor is heated using a heating mantle equipped with thermocouples. After the reaction, the catalyst is filtered off and the composition of the liquid fraction is analyzed by GCMS. The control reactions with all three substrates, but no MOF catalyst present were also performed and consistently showed $0 \%$ conversion (see Table S1).

Characterization methods. ${ }^{1} \mathrm{H}$ and ${ }^{13} \mathrm{C}$ NMR experiments were performed on a Varian 500 $\mathrm{MHz}$ spectrometer in $\mathrm{d}_{10}$-xylene. GC-MS analysis was performed on an Agilent Varian CP3800 Gas Chromatograph using a DB-WaxETR column $(30 \mathrm{~m} \times 0.25 \mathrm{~mm} \times 0.5 \mu \mathrm{m})$ with output even split between a Saturn 2000R mass-spectrometer and an FID detector $\left(\mathrm{H}_{2} /\right.$ air $)$. Helium was used as a carrier gas, with a constant column flow of $1.2 \mathrm{~mL} / \mathrm{min}$. The column temperature control was adapted from the method detailed in Molinari et al. ${ }^{3}$ FID quantitation of the mass balance of products and reagents was achieved using individual and mixed standards with linear regression analysis of the integrated peak intensities. The MOF catalyst powders were characterized using scanning electron microscopy (SEM), X-ray diffraction (XRD), and energy dispersive X-ray spectroscopy (EDS). The powders were analyzed using a JEOL 7600 microscope (JEOL Ltd., Tokyo, Japan) operating at $15 \mathrm{kV}$. The XRD patterns were recorded on a PANalytical Empyrean X-ray diffractometer equipped with a PIXcel ${ }^{3 \mathrm{D}}$ detector and operated at $45 \mathrm{kV}$ and $40 \mathrm{~mA}$ using $\mathrm{Cu} K \alpha$ radiation $(\lambda=1.5418 \AA$ ). Scattering intensities were measured using the Bragg-Brentano $(\theta-2 \theta)$ geometry with a step size of $0.026^{\circ}$. Surface area measurements (Brunauer, Emmett and Teller (BET) method) were determined using a Micromeritics ASAP 2020 porosimeter. The composition in the gas phase in entry above the reaction was monitored by Residual Gas Analyzer RGA-100 from Stanford Research Systems. A minimal amount of gas sample in the reaction vessel was bled into the RGA at room temperature by a flow-restricted valve to ensure the gas pressure in RGA is below $5 \times 10^{-4} \mathrm{~Pa}$, as required by the RGA instrument. Inductively coupled plasma optical emission spectrometry (ICP-OES) analysis was performed by ALS Environmental, Inc. For x-ray photoelectron spectroscopy (XPS) characterization, samples were pressed on pure indium foil substrate and then illuminated with an Al K $\alpha$ source (Omicron model DAR400) using photons of $1490 \mathrm{eV}$. Photoelectrons were detected using a Physical Electronics model 10-360 electron energy analyzer. The slight charging resulting from the poor electrical conductivity of the samples was corrected by adjusting the binding energy of the most prominent $\mathrm{C} 1 \mathrm{~s}$ peak to $284.8 \mathrm{eV}$. Peaks were fitted using CasaXPS software. Shirley-type background subtraction was used in all fits. 


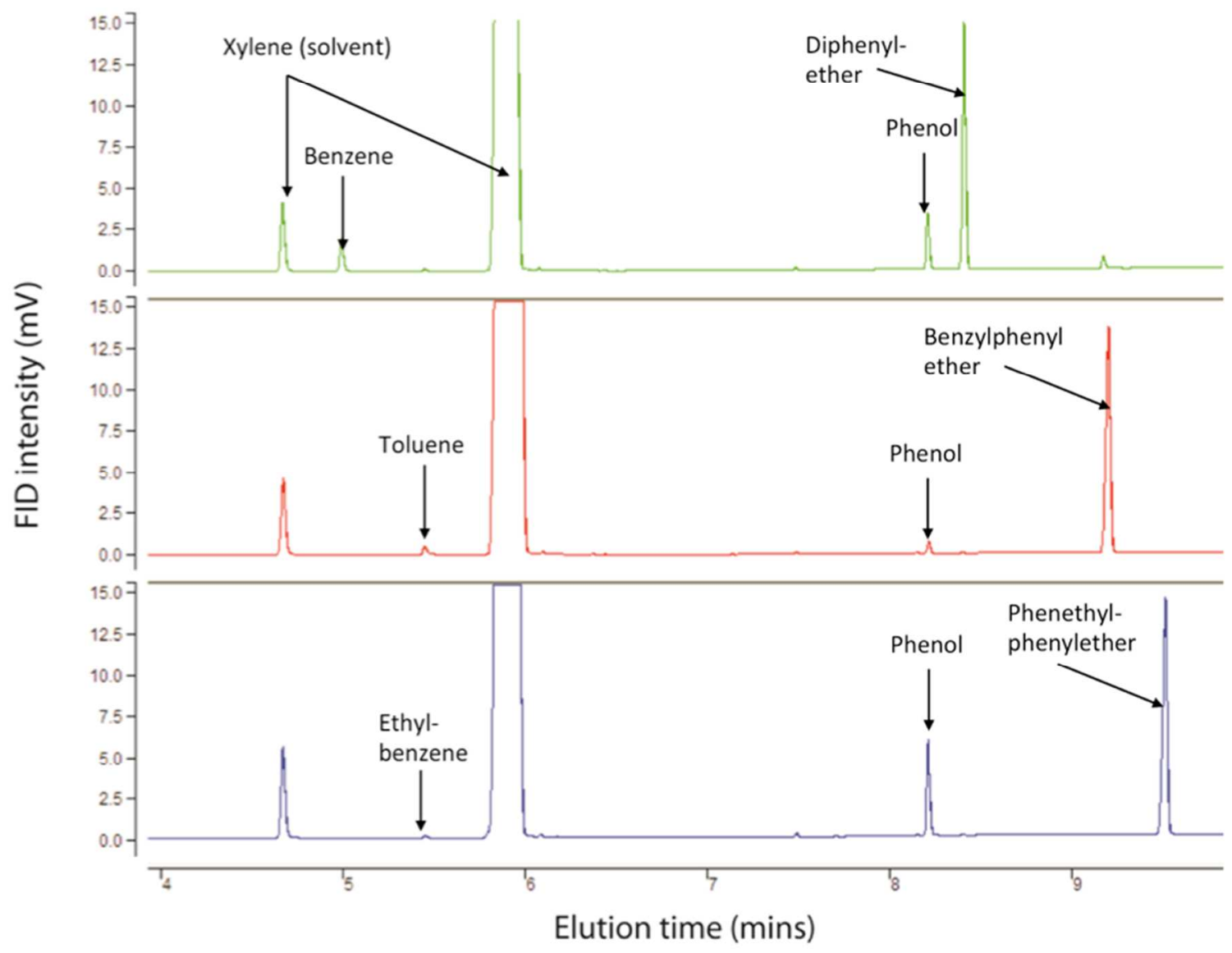

Figure S1. Gas chromatograms showing partial conversion of the substrates into the corresponding hydrocarbon and phenol at $120{ }^{\circ} \mathrm{C}$ under 10 bar hydrogen in the presence of Ni@IRMOF-74(II). 


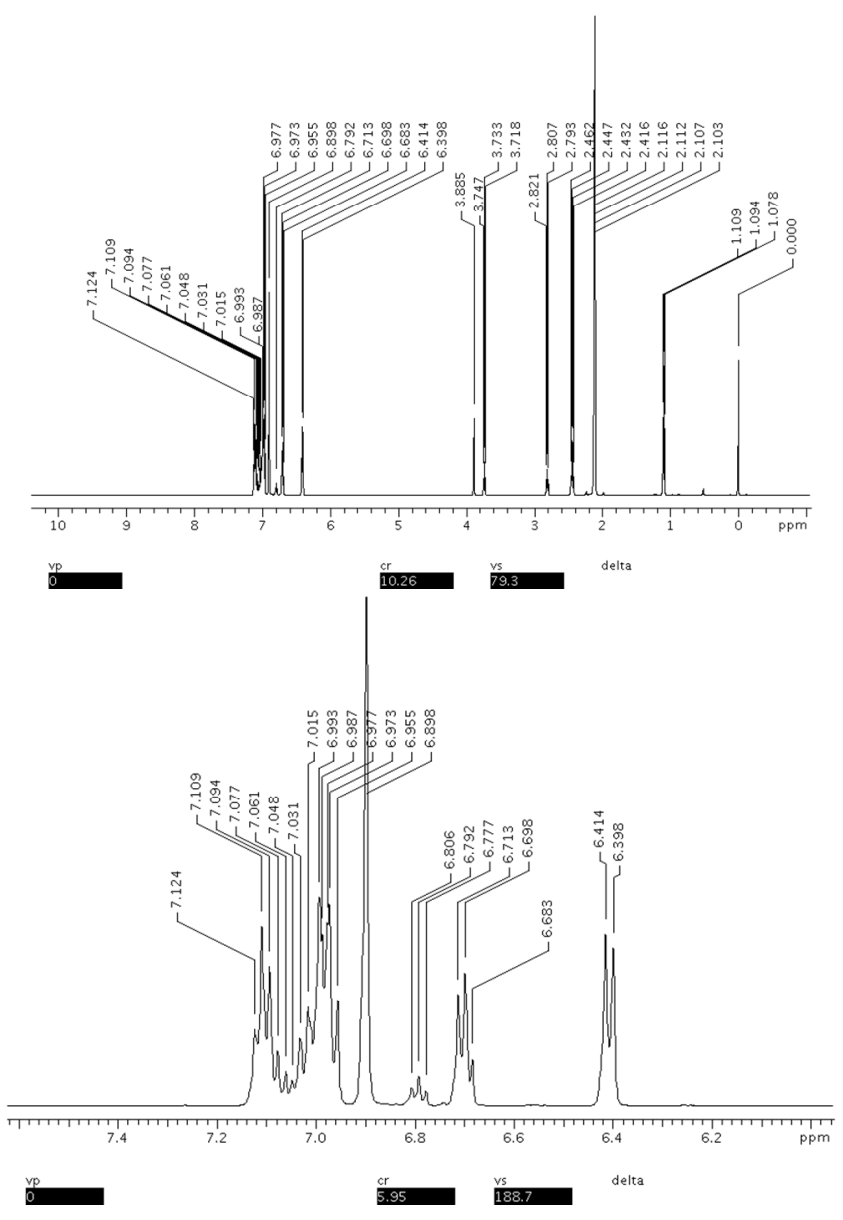

Figure S2. $\quad{ }^{1} \mathrm{H}$ NMR results showing partial conversion of PPE into ehtylbenzene and phenol at $120{ }^{\circ} \mathrm{C}$ under 10 bar hydrogen in the presence of Ni@IRMOF-74(II) in $\mathrm{d}_{10^{-}}$ xylene. 


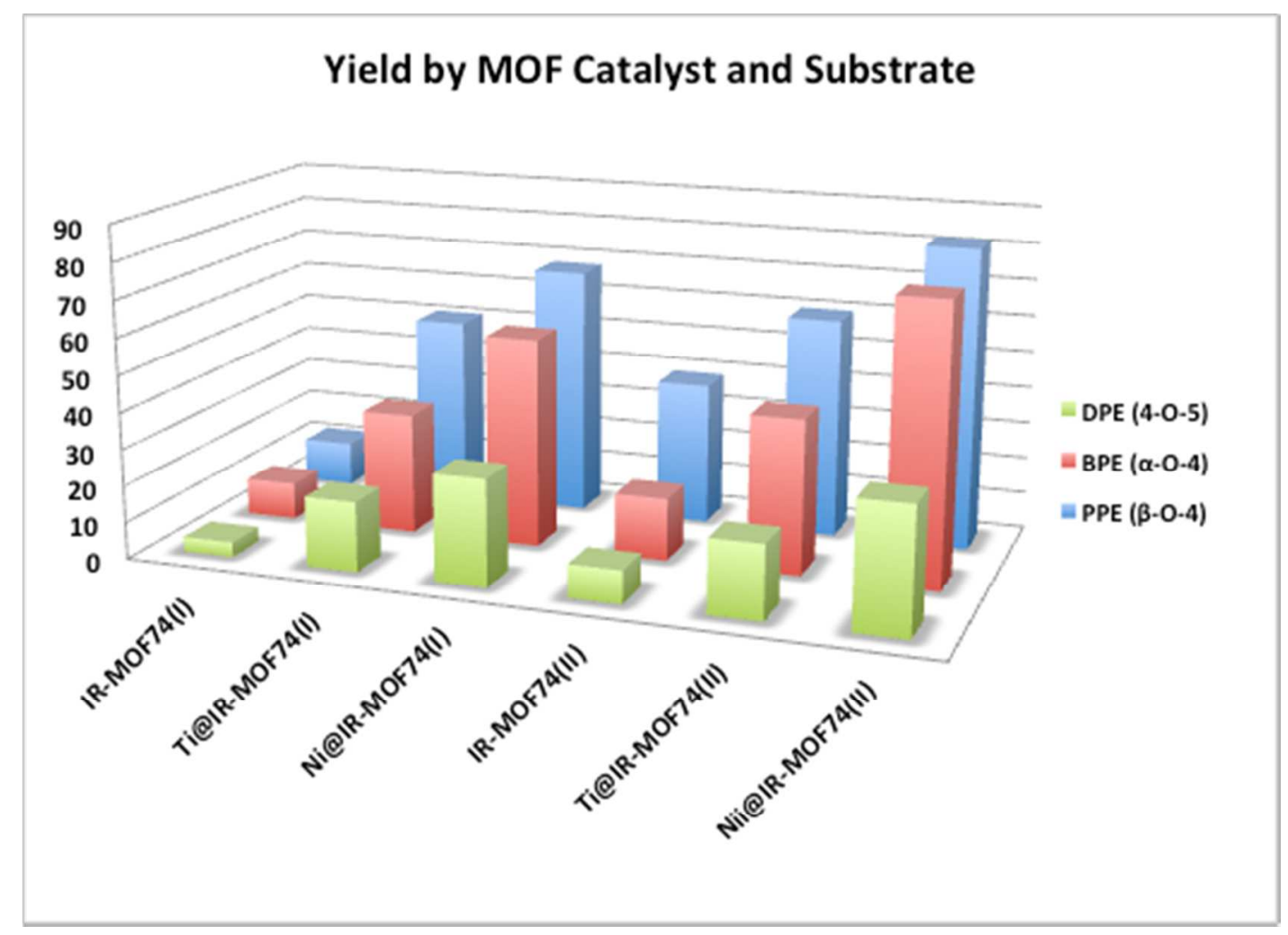

Figure S3. Conversion efficiencies of the substrates into the corresponding hydrocarbon and phenol at $120^{\circ} \mathrm{C}$ under 10 bar hydrogen in the presence of Ni@IRMOF-74(II).

Table S1. Catalytic effect of Ni@IRMOF-74(I) upon cycling (cycles 2 through 5 are shown here; cycle 1 is entry 3 in Table 1), as well as results of tests in the absence of catalyst for all three substrates ( $1=$ ethylbenzene, $2=$ toluene, $3=$ benzene, $4=$ phenol).

\begin{tabular}{|c|c|c|c|c|c|c|c|c|c|}
\hline \multirow{2}{*}{ Entry } & \multirow{2}{*}{ Catalyst } & \multirow{2}{*}{ Substrate } & \multirow{2}{*}{$\mathrm{T},{ }^{\circ} \mathrm{C}$} & \multirow{2}{*}{$\begin{array}{l}\text { Time, } \\
\text { Hours }\end{array}$} & \multirow{2}{*}{$\begin{array}{c}\text { Conv } \\
\%\end{array}$} & \multicolumn{4}{|c|}{ Selectivity } \\
\hline & & & & & & 1 & 2 & 3 & 4 \\
\hline 26 & $\begin{array}{c}\text { Ni@IRMOF-74(I) } \\
\text { (Cycle 2) }\end{array}$ & PPE $(\beta-O-4)$ & 120 & 16 & 69 & 93 & & & 95 \\
\hline 27 & $\begin{array}{c}\text { Ni@IRMOF-74(I) } \\
\text { (Cycle 3) }\end{array}$ & PPE ( $\beta-O-4)$ & 120 & 16 & 68 & 92 & & & 94 \\
\hline 28 & $\begin{array}{c}\mathrm{Ni@IRMOF-74(I)} \\
\text { (Cycle 4) }\end{array}$ & PPE ( $\beta-O-4)$ & 120 & 16 & 65 & 90 & & & 93 \\
\hline 29 & $\begin{array}{c}\mathrm{Ni@IRMOF-74(I)} \\
\text { (Cycle 5) }\end{array}$ & PPE $(\beta-O-4)$ & 120 & 16 & 67 & 91 & & & 94 \\
\hline 30 & No catalyst & PPE $(\beta-O-4)$ & 120 & 16 & 0 & 0 & & & 0 \\
\hline 31 & No catalyst & $\operatorname{BPE}(\alpha-O-4)$ & 120 & 16 & 0 & & 0 & & 0 \\
\hline 32 & No catalyst & DPE (4-O-5) & 120 & 16 & 0 & 0 & & & 0 \\
\hline
\end{tabular}




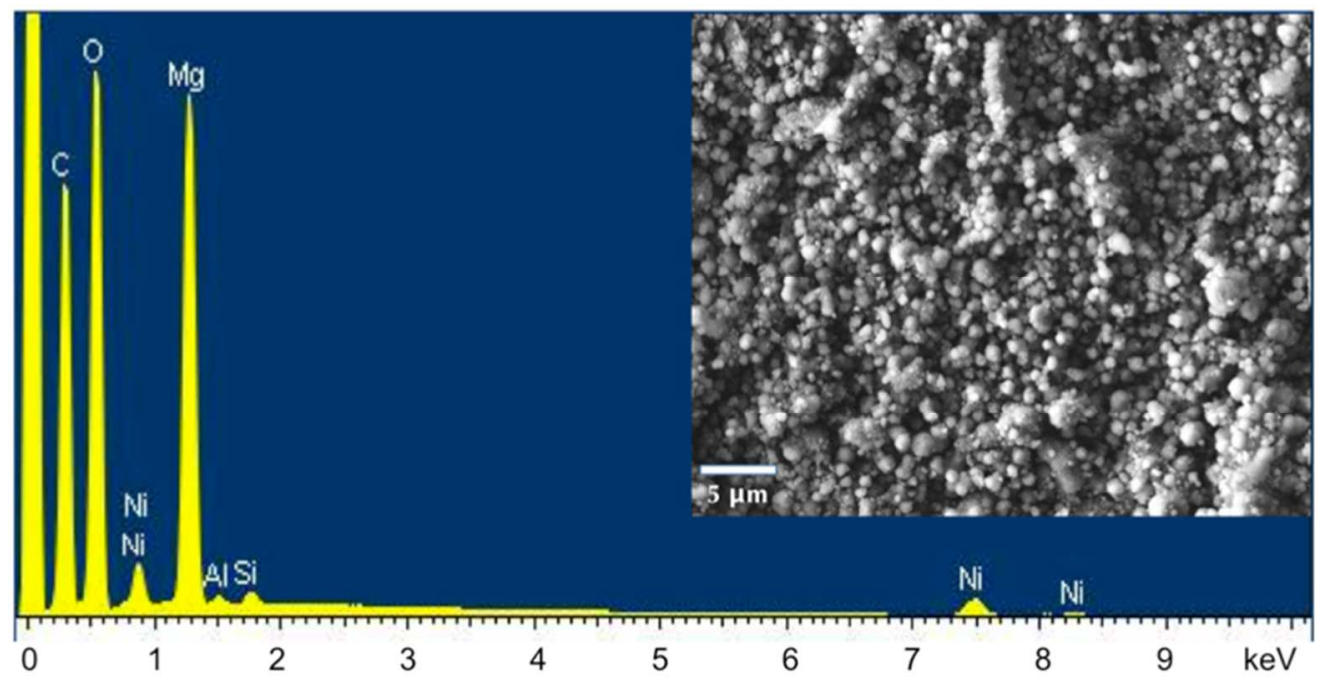

Figure S4. A) EDS spectrum of Ni@IRMOF-74(I); the inset shows a representative SEM image of the as-synthesized catalyst sample. The small aluminum and silicon peaks are from the sample holder; 
XPS spectra. Figure S5(A) shows the overall XPS spectrum of the Ti@IRMOF-74(I) sample with characteristic $\mathrm{Mg}, \mathrm{C}$, and $\mathrm{O}$ peaks from the MOF, as well as small Ti features from the dopant. Figure S5(B) shows the entire XPS spectrum of Ni@IRMOF-74(I), again with the characteristic $\mathrm{Mg}, \mathrm{C}$, and $\mathrm{O}$ peaks from the MOF, as well as a small Ni contribution from the dopant. The Ti 2p XPS spectra from Ti@IRMOF-74(I) exhibit two prominent peaks centered at $457.5 \mathrm{eV}\left(\mathrm{Ti} 2 \mathrm{p}_{3 / 2}\right)$ and $463.0 \mathrm{eV}\left(\mathrm{Ti} 2 \mathrm{p}_{1 / 2}\right)$ characteristic of Ti(III)Cl ${ }_{3}{ }^{4}$ Figure S5(C) shows the characteristic Ni 2p features in the Ni@IRMOF-74(I) sample after exposure to air for 15 (minimum amount of time required to transfer the sample to the XPS chamber), 30 and 300 seconds. Figure S5(C) (left panel) shows two prominent Ni $2 \mathrm{p}_{3 / 2}$ peaks are located at $852.6 \mathrm{eV}$ and $856.3 \mathrm{eV}$, corresponding to metallic $\mathrm{Ni}^{0}(45 \%)$ and $\mathrm{Ni}(\mathrm{OH})_{2}(55 \%)$, respectively. ${ }^{5,6}$ The quantification of the spectrum collected after 30 second exposure to air (middle panel) reveals that $70 \%$ of the Ni species near the surface (region probed by XPS) is in the $\mathrm{Ni}(\mathrm{OH})_{2}$ phase, while $30 \%$ is metallic Ni. This is the result of metallic Ni nanoparticles oxidizing in air even after only 15 or 30 seconds of exposure, which is fully consistent with the literature on $\mathrm{Ni}$ nanoparticles. ${ }^{7}$ To verify the effect of air exposure, we prepared another identical Ni@IRMOF74(I) sample that was exposed to air for 300 seconds (Figure S5(C), right panel), which clearly shows that all of the nickel is converted to $\mathrm{Ni}(\mathrm{OH})_{2}$. No other Ni oxides were detected.
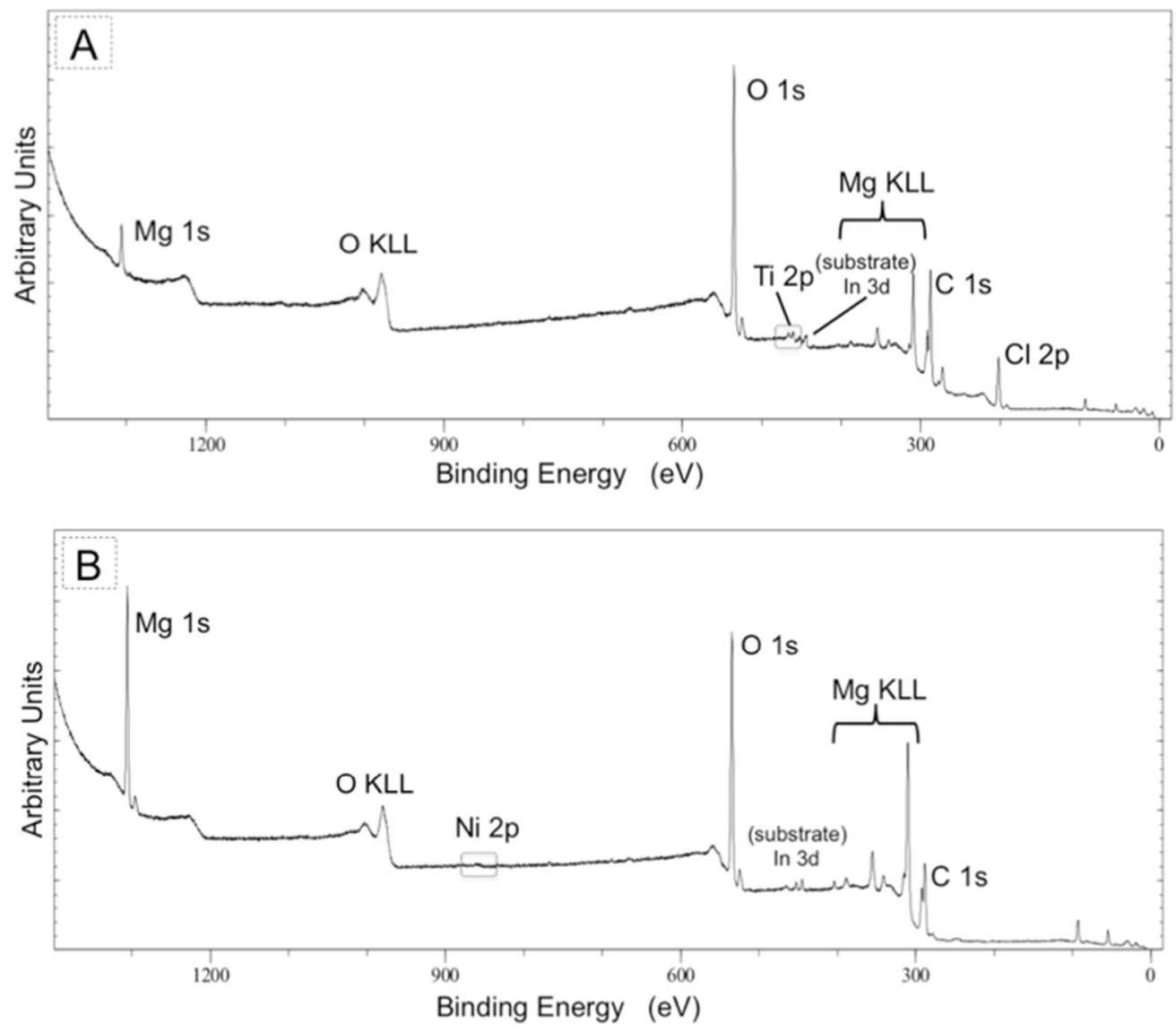

Figure S5. XPS spectra. (A) Ti@IRMOF-74(I); B) Ni@IRMOF-74(I). 


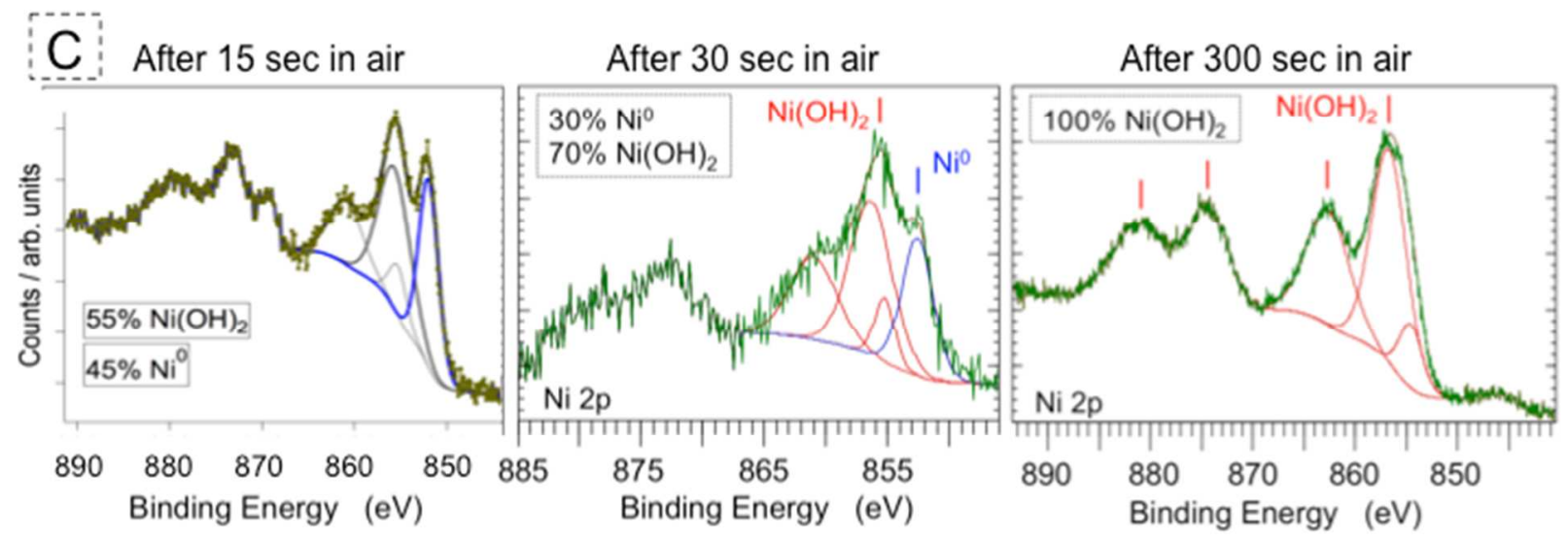

Figure S5(C). Expanded Ni 2p XPS features. The XPS spectra for Ni@IRMOF-74(I) samples were taken after 15,30 and $300 \mathrm{sec}$ exposure to air. The data suggests that a significant amount of $\mathrm{Ni}$ in the MOF is in oxidation state (0).

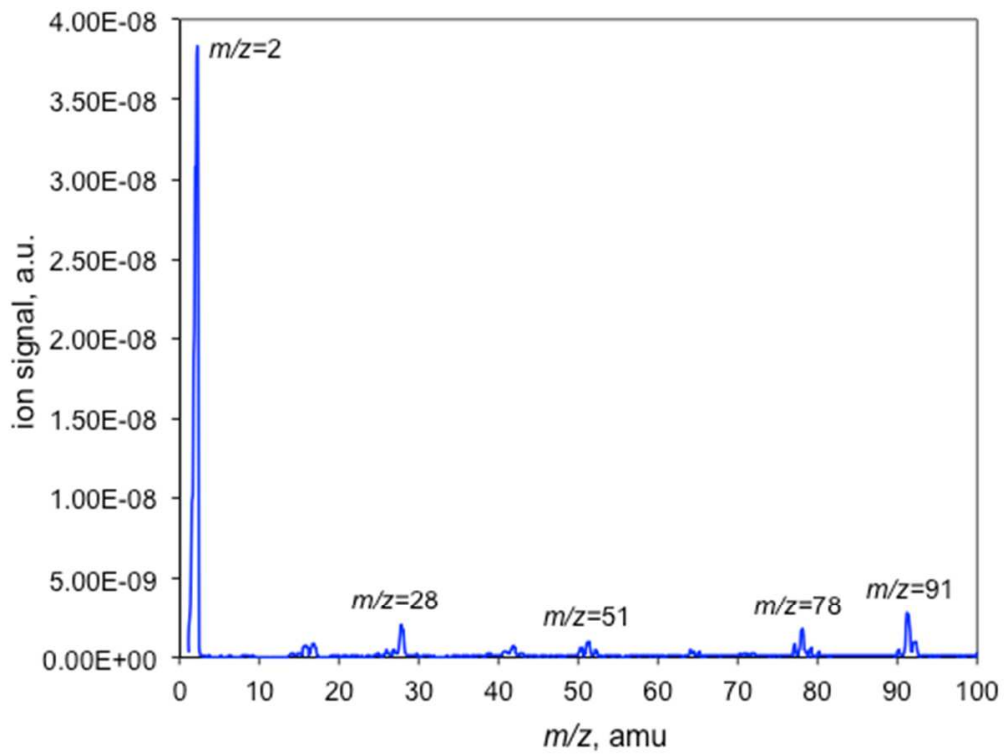

Figure S6. Residual Gas Analysis of the volatiles from reaction entry \#6. The most significant feature at $m / z=2$ corresponds to hydrogen; the species at $m / z=78$ and 91 correspond to fragments of aromatic hydrocarbons $\left(\mathrm{C}_{6} \mathrm{H}_{6}{ }^{+}\right)$and $\left.\mathrm{C}_{7} \mathrm{H}_{8}{ }^{+}\right)$from the solvent and/or from reaction products. 


\section{Computational Methods}

Geometries. The geometries of phenylethylphenyl ether, benzylphenyl ether, and diphenyl ether representing the $\beta-\mathrm{O}-4, \alpha-\mathrm{O}-4$, and 4-O-5 linkages in natural lignin, respectively, were optimized using the M06-2X hybrid exchange-correlation functional and the $6-31+\mathrm{G}(\mathrm{d}, \mathrm{p})$ basis set. Optimized structures and C-O bond distances are given in Table S2. The geometries of all product species were also optimized. Vibrational frequencies were computed to verify that the computed structures corresponded to energy minima; no imaginary frequencies were found. Bond dissociation energies (BDEs) for the ether bonds (C-O) were determined from the difference of the sum of the energies of the dissociated product fragments and the energy of the molecule:

$$
\mathrm{BDE}=\Delta H_{\mathrm{BDE}}^{\circ}(T)=\left(E_{\mathrm{Frag} 1}+E_{\mathrm{Frag} 2}\right)-E_{\mathrm{Mol}}
$$

where $E_{\mathrm{Mol}}$ is the total energy of the molecule and $E_{\mathrm{Frag} 1}$ and $E_{\mathrm{Frag} 2}$ are the energies of the products resulting from cleavage of the $\mathrm{C}-\mathrm{O}$ linkages, corrected to $393 \mathrm{~K}$ by including the zeropoint energies (ZPE) and thermal contributions obtained from the computed frequencies.

Cluster model. To assess the interaction between model compound and the MOFs, we simulated Mg-IRMOF-74(I) by a cluster model comprised of four $\mathrm{Mg}^{2+}$ ions coordinated to five oxygen donor atoms from the linkers. This approximates one side of the core unit of hexagonal pore (Figure S7). The initial atom positions were taken from the experimental crystal structures ${ }^{1,2}$ for IRMOF-74(I) (ref. code VOGTIV) and IRMOF-74(II) (ref. code RAVVUH). The cluster was then terminated by $-\mathrm{H},-\mathrm{CH}_{3}$, and $\mathrm{Li}$, following the method of $\mathrm{Yu}$ et al. ${ }^{8}$ Cluster geometries, with and without the aromatic ether compounds, were then relaxed without any constraints and the binding energies computed from the relaxed geometries, using the hybrid QM/QM method at the MO6-2x/6-31G(d,p):PM6 levels of theory. For these calculations, the ONIOM ("Our own $N$-layered Integrated molecular Orbital and Molecular mechanics") approach implemented in Gaussian09 was used. ${ }^{9}$ During optimization of the various MOF-model compound complexes, the model compounds were treated at the MO6$2 \mathrm{X} / 6-31+\mathrm{G}(\mathrm{d}, \mathrm{p})$ level of theory, whereas the MOF cluster was treated at the semi-empirical PM6 level of theory. The binding energies were then obtained at the MO6-2X/6-31+G(d,p) level of theory. The effects of solvent on the binding energies were computed using the integral equation formalism of the polarizable continuum model (IEF-PCM) into the self-consistent reaction field, where the solvent (xylene) is taken into account by means of a polarizable dielectric medium. Basis set superposition errors were removed in all binding energy calculations using the counterpoise method. ${ }^{10}$

Charge transfer analysis. According to $\mathrm{DFT}^{11}$, the chemical potential $(\mu)$ and chemical hardness $(\eta)$ are defined as:

$$
\begin{gathered}
\chi=-\mu=-\left(\frac{\partial E}{\partial N}\right)_{v(\vec{r})} \\
\eta=\frac{1}{2}\left(\frac{\partial^{2} E}{\partial N^{2}}\right)_{v(\vec{r})}=\frac{1}{2}\left(\frac{\partial \mu}{\partial N}\right)_{v(\vec{r})}
\end{gathered}
$$


where $E$ is the total energy of the system, $N$ is the number of electrons in the system and $v(\vec{r})$ is the external potential. $\mu$ is identified as the negative of the electronegativity $(\chi)$. By applying finite difference approximation to Eqs. (2) and (3) we get the operational definition for $\eta$ and $\mu$ as:

$$
\begin{gathered}
\mu=-\frac{(I P+E A)}{2} \\
\eta=\frac{I P-E A}{2}
\end{gathered}
$$

Chemical potential and chemical hardness can be rewritten using Koopmans' theorem in terms of the vertical ionization potential (IP) and electron affinity (EA) as,

$$
\begin{aligned}
& \eta=\frac{E_{L U M O}-E_{H O M O}}{2} \\
& \mu=\frac{E_{L U M O}+E_{H O M O}}{2}
\end{aligned}
$$

where $\mathrm{E}_{\mathrm{LUMO}}$ is the lowest unoccupied molecular orbital's energy and $\mathrm{E}_{\text {HOMO }}$ is the highest occupied molecular orbital's energy.

The global interactions between the MOF cluster model and aromatic ether model compounds were determined using the quantity $\Delta N$, which represents the fractional number of electrons transferred from a system $\mathrm{A}$ to a system $\mathrm{B}$, given by: ${ }^{12}$

$$
\Delta N=\frac{\mu_{B}-\mu_{A}}{2\left(\eta_{A}+\eta_{B}\right)}
$$

We can also predict the flow of electrons using Sanderson's electronegativity equalization principle, ${ }^{13}$ which states that there will be a flow of electrons from lower electronegativity (higher chemical potential) to that of higher electronegativity (lower chemical potential) until the electronegativity values are equalized to a value roughly equal to the geometric mean of the individual electronegativities i.e. electron will flow from molecule $\mathrm{B}$ to molecule $\mathrm{A}$ if $\chi_{A}>\chi_{B}$ where $\chi_{A}$ and $\chi_{B}$ are the electronegativities of the molecules $\mathrm{A}$ and $\mathrm{B}$ respectively. 


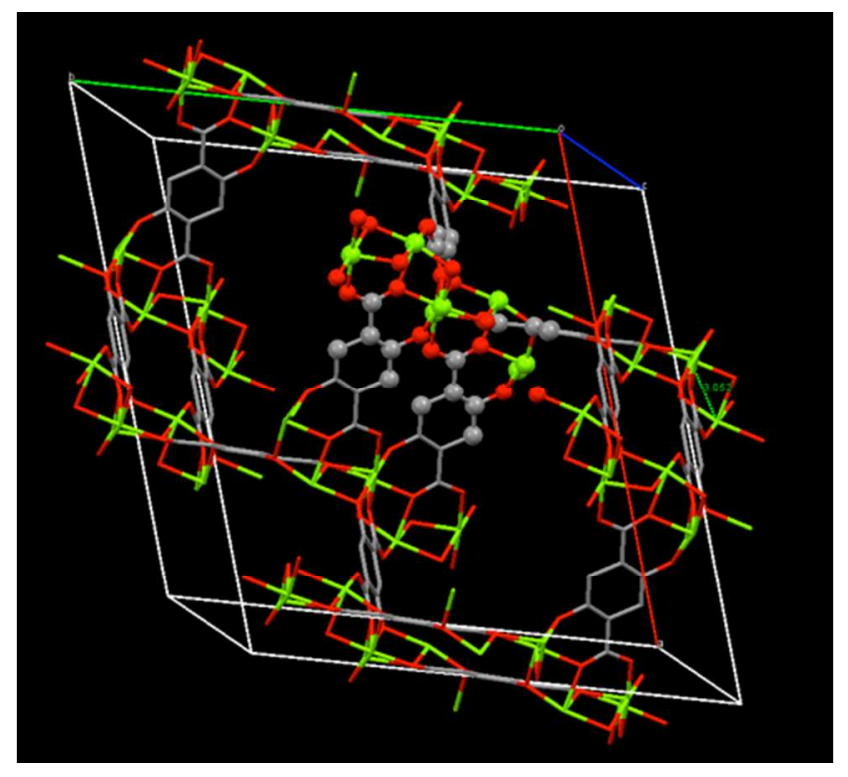

Figure S7: Structure of IRMOF-74(I) cluster consisting of four metal ions coordinated to linkers, which approximates one side of the core unit of the hexagonal pore. Hydrogen atoms are omitted for clarity. Atom color code: C: gray; O: red; Mg: green. 
Substrate accessibility to MOF pores. We determined that the pores of the two MOFs are large enough to accommodate the all three aromatic ethers considered in this study, using the experimentally determined crystal structures. ${ }^{1,2}$ Figure S8 illustrates the pore size diameter of IRMOF-74(I) and IRMOF-74(II). The longest intramolecular distance in the optimized phenylethylphenyl ether, benzylphenyl ether and diphenyl ether geometries corresponds to a straight line drawn between the blue-highlighted atoms molecular structures shown in Figure S7. These distances range between $9 \AA$ and $14 \AA$; the substrates are therefore small enough to fit into the pores of both IRMOF-74(I) and IRMOF-74(I).

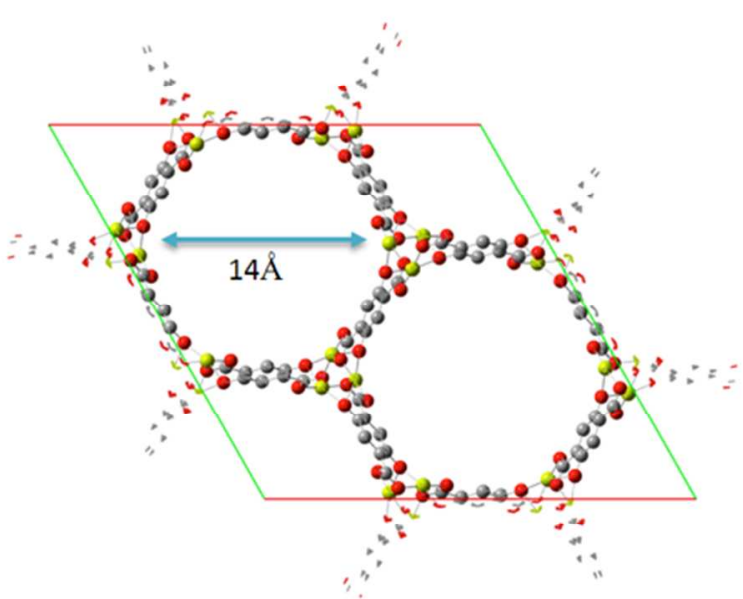

IRMOF-74-I

VOGTIV
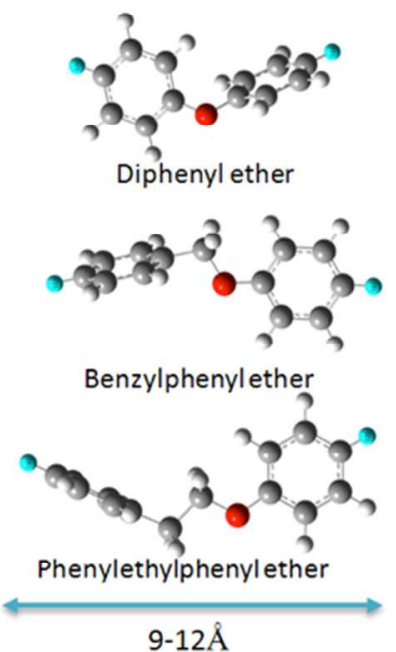

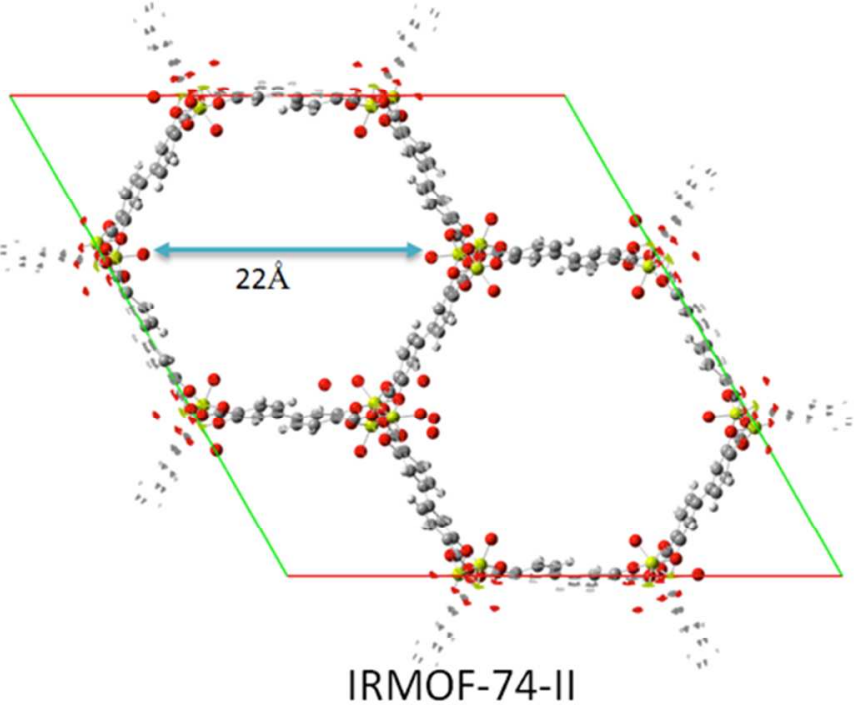

IRMOF-74-II

RAVVUH
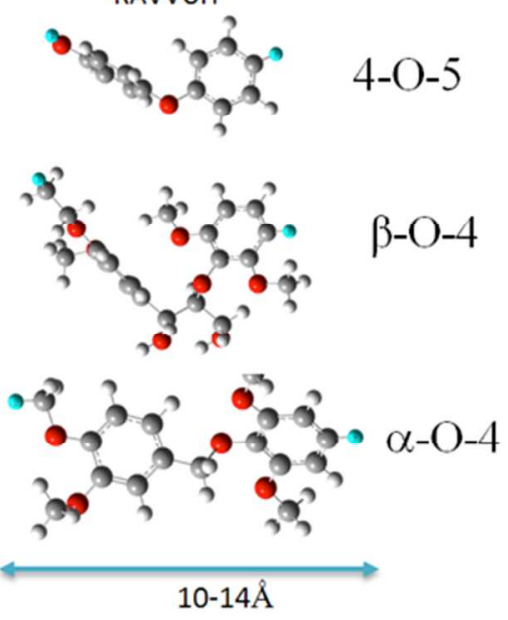

Figure S8. Top: pore diameters of IRMOF-74(I) and IRMOF-74(II). Bottom: largest intramolecular distances in the aromatic ether compounds, corresponding to a straight line between the blue-highlighted atoms in the optimized geometries. 
Reaction energies. The calculated gas-phase ether bond dissociation energies at $393 \mathrm{~K}$ are given in Table S2 and the calculated stabilization energies of the reactants $\left(\mathrm{H}_{2}+\right.$ Subtrates $)$ and products (hydrocarbon + phenol) in the presence of IRMOF-74(I) cluster model are shown in Table S3. Figure S9 shows configurations of aromatic ether compounds with the cluster model, determined by the $\mathrm{QM} / \mathrm{QM}$ method described above. Among the three ether bond linkage types, diphenylether (4-O-5) has the shortest C-O bond distance $(1.37 \AA$ ); the C-O bond distances in benzylphenylether ( $\beta-\mathrm{O}-4)$ and phenylethylphenyl ether $(\alpha-\mathrm{O}-4)$ are $1.41 \AA$ and $1.42 \AA$, respectively. The computed BDE indicate that the ether linkages in the $\alpha-\mathrm{O}-4$ and $\beta-\mathrm{O}-4$ model compounds are weaker than the 4-O-5 linkages, as reported previously. ${ }^{7}$

Table S2. Optimized geometries and calculated gas-phase bond dissociation energies (BDE) at $393 \mathrm{~K}$ of the ether linkages in the aromatic ether model compounds.

\begin{tabular}{|c|c|c|}
\hline $\begin{array}{l}\text { Aromatic ether } \\
\text { compounds }\end{array}$ & Optimized structure & $\mathrm{BDE}$ in $\mathrm{kJ} / \mathrm{mol}$ \\
\hline $\begin{array}{l}\text { phenylethylphenyl } \\
\text { ether (PPE) }\end{array}$ & & 290.8 \\
\hline $\begin{array}{l}\text { benzylphenyl } \\
\text { ether (BPE) }\end{array}$ & & 237.7 \\
\hline $\begin{array}{l}\text { diphenyl ether } \\
\text { (DPE) }\end{array}$ & & 345.2 \\
\hline
\end{tabular}


Table S3. Calculated binding energies $\left(\Delta H^{\circ}{ }_{\mathrm{B}}(T) ; \mathrm{kJ} / \mathrm{mol}\right)$ at $393 \mathrm{~K}$ of the reactants $\left(\mathrm{H}_{2}+\right.$ model compound) and products (hydrocarbon + phenol) on the IRMOF-74 cluster model, relative to gas phase and in the presence of xylene solvent environment. The optimized geometry of $\left(\mathrm{H}_{2}+\right.$ model compound $)$ with the cluster was used to obtain the "gas phase" results. Results accounting for a xylene solvent environment were obtained by performing a single point calculation using the geometry used for the gas-phase calculation.

\begin{tabular}{|l|c|c|c|c|}
\hline \multirow{3}{*}{ Substrate } & \multicolumn{2}{|c|}{$\begin{array}{c}\text { Reactants } \\
\left(\mathrm{H}_{2}+\text { model compound) }\right.\end{array}$} & \multicolumn{2}{c|}{$\begin{array}{c}\text { Reaction products } \\
\text { (hydrocarbon + phenol) }\end{array}$} \\
\cline { 2 - 5 } & Gas phase & $\begin{array}{c}\text { Solvent } \\
\text { (Xylene) }\end{array}$ & Gas phase & $\begin{array}{c}\text { Solvent } \\
\text { (Xylene) }\end{array}$ \\
\hline PPE & 137.4 & 81.2 & 152.8 & 97.1 \\
\hline BPE & 119.5 & 70.1 & 178.9 & 118.6 \\
\hline DPE & 47.6 & 34.7 & 55.7 & 36.4 \\
\hline
\end{tabular}
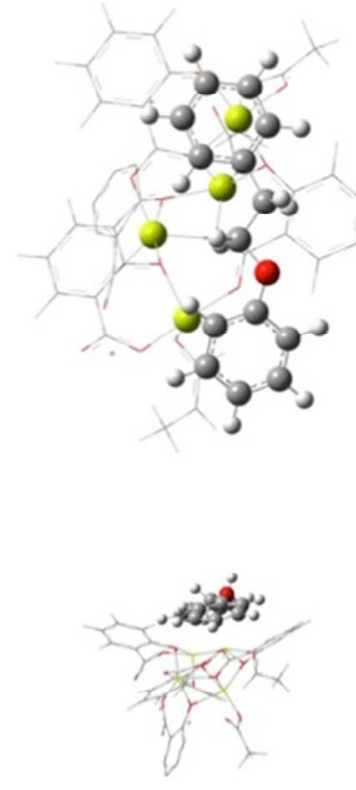

PPE
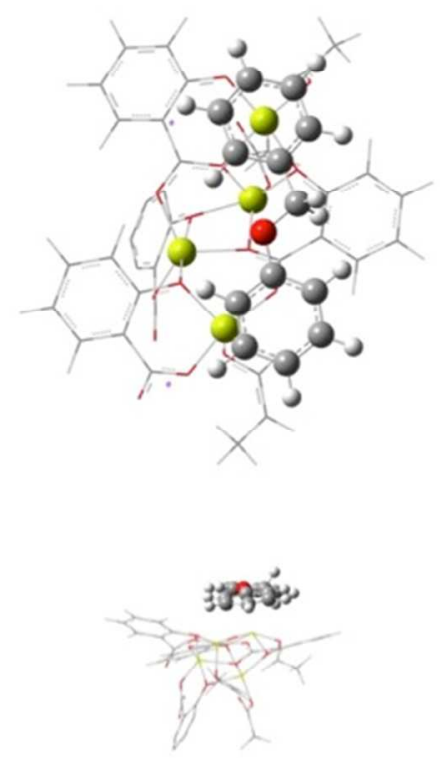

BPE
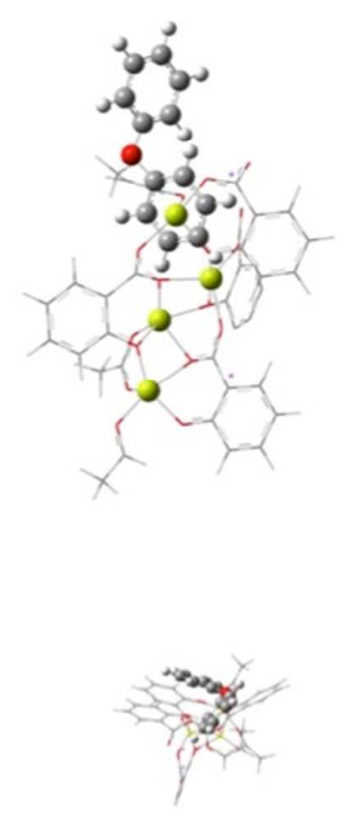

DPE

Figure S9. Optimized geometries of substrate-MOF cluster units (top and side views) using QM/QM method. Aromatic ether compounds and the OMS (yellow spheres) of MOF cluster unit are highlighter for clarity. Atom code: $\mathrm{Mg}$ (yellow); $\mathrm{O}$ (red); C(black); H(white). 
Molecular electrostatic potential map. The MESP map (Figure S10) for each aromatic ether compound in the gas phase indicates the distinct separation of positively and negatively charged regions in these molecules, which will we expect will plate a dominant role in interactions with the MOF. Comparison with the optimized cluster-substrate geometries shown in Figure S8 indicates that the negative regions located on the rings are localized on the electron-accepting $\mathrm{Mg}^{2+}$ open metal sites of the MOF cluster, whereas the negatively charged $\mathrm{O}$ atoms do not interact directly with the metal ions, probably for steric reason. However, this makes them accessible for electrophilic attack by $\mathrm{H}_{2}$.
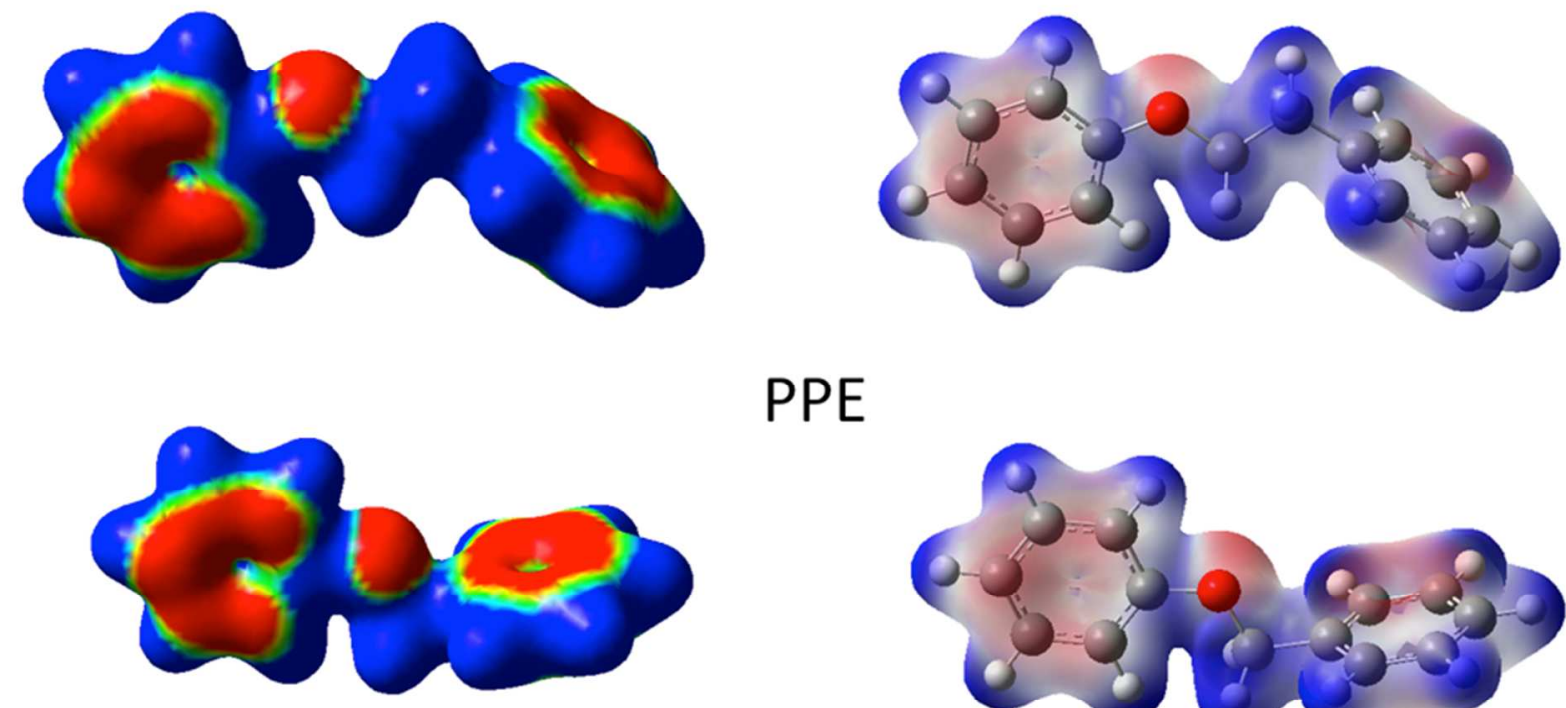

\section{PPE}

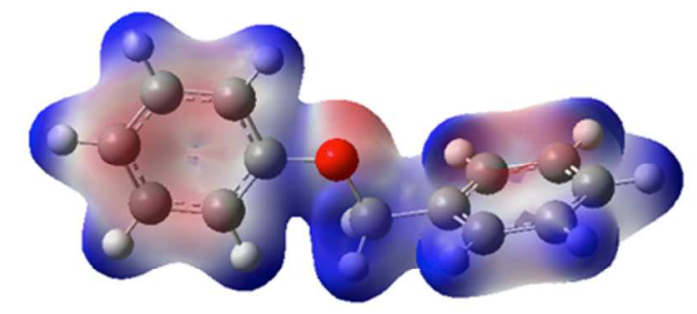

\section{BPE}
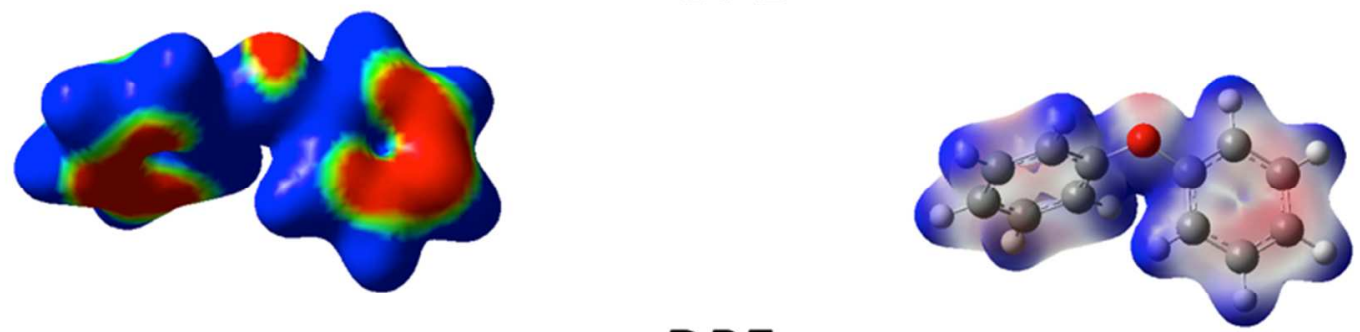

\section{DPE}

Figure S10. Molecular electrostatic potential map (MESP) of aromatic ether compounds (in the absence of the MOF-74 cluster) at the \pm 0.02 au isosurface. The color scale indicates the charges on the atoms: red $=$ most negative, green $=$ neutral, blue $=$ most positive charge. 
Charge Transfer Analysis $(\Delta \mathbf{N})$. The interactions between the selected aromatic ether compounds (A) and the MOF cluster (B) was also quantified by using the quantity $\Delta N$ representing the fractional number of electrons transferred from A to B. Values of $\Delta N$ are presented in Tables S4. In general, electrons flow from less electronegative regions to more electronegative ones. Combining this fact with the definition of $\Delta N$ (Equation 8) shows that charge transfer values are negative for aromatic ether compounds, indicating that they are electron donors. In the interaction of the MOF cluster with aromatic model compounds, the MOF accepts the maximum charge from PPE, followed by BPE and DBE. This trend is consistent with the computed interaction energies of the model compounds with the MOF cluster, as well as with the observed trend in conversion.

Table S4. Charge Transfer $(\Delta \mathrm{N})$ between aromatic ether compounds and MOF, computed from Equation 10.

\begin{tabular}{|c|c|c|c|}
\hline & $\begin{array}{c}\text { Chemical hardness }(\eta) \\
\text { in eV }\end{array}$ & $\begin{array}{c}\text { Chemical potential }(\mu) \\
\text { in eV }\end{array}$ & $\Delta \mathrm{N}$ \\
\hline PPE & 3.90 & -3.63 & -0.040 \\
\hline BPE & 3.88 & -3.66 & -0.038 \\
\hline DPE & 3.82 & -3.70 & -0.034 \\
\hline MOF Cluster Model & 1.84 & -4.09 & \\
\hline
\end{tabular}




\section{References:}

1. Deng, H.; Grunder, S.; Cordova, K. E.; Valente, C.; Furukawa, H.; Hmadeh, M.; Gándara, F.; Whalley, A. C.; Liu, Z.; Asahina, S.; Kazumori, H.; O’Keeffe, M.; Terasaki, O.; Stoddart, J. F.; Yaghi, O. M. Science 2012, 336, 1018-1023.

2. Caskey, S. R.; Wong-Foy, A. G.; Matzger, A. J. J. Am. Chem. Soc. 2008, 130, 10870-10871.

3. Molinari, V.; Giordano, C.; Antonietti, M.; Esposito, D. J. Am. Chem. Soc. 2014, 136, 1758-1761.

4. Mousty-Desbuquoit, C.; Riga, J.; Verbist, J. J. Inorg. Chem. 1987, 26, 1212-1217.

5. Biesinger, M. C.; Payne, B. P.; Grosvenor, A. P.; Lau, L. W. M.; Gerson, A. R.; Smart, R. S. C. Appl. Surface Sci. 2011, 257, 2717-2730.

6. Biesinger, M. C.; Lau, L. W. M.; Gerson, A. R.; Smart, R. S. C. Phys. Chem. Chem. Phys. 2012, 14, 2434-2442.

7. Phung, X.; Groza, J.; Stach, E. A.; Williams, L. N.; Ritchey, S. B. Mater. Sci. Eng.: A 2003, 359, 261-268.

8. Yu, D.; Yazaydin, A. O.; Lane, J. R.; Dietzel, P. D. C.; Snurr, R. Q. Chem. Sci. 2013, 4, 3544-3556.

9. Gaussian 09, R. A., Frisch, M. J.; Trucks, G. W.; Schlegel, H. B.; Scuseria, G. E.; Robb, M. A.; Cheeseman, J. R.; Scalmani, G.; Barone, V.; Mennucci, B.; Petersson, G. A.; Nakatsuji, H.; Caricato, M.; Li, X.; Hratchian, H. P.; Izmaylov, A. F.; Bloino, J.; Zheng, G.; Sonnenberg, J. L.; Hada, M.; Ehara, M.; Toyota, K.; Fukuda, R.; Hasegawa, J.; Ishida, M.; Nakajima, T.; Honda, Y.; Kitao, O.; Nakai, H.; Vreven, T.; Montgomery, Jr., J. A.; Peralta, J. E.; Ogliaro, F.; Bearpark, M.; Heyd, J. J.; Brothers, E.; Kudin, K. N.; Staroverov, V. N.; Kobayashi, R.; Normand, J.; Raghavachari, K.; Rendell, A.; Burant, J. C.; Iyengar, S. S.; Tomasi, J.; Cossi, M.; Rega, N.; Millam, N. J.; Klene, M.; Knox, J. E.; Cross, J. B.; Bakken, V.; Adamo, C.; Jaramillo, J.; Gomperts, R.; Stratmann, R. E.; Yazyev, O.; Austin, A. J.; Cammi, R.; Pomelli, C.; Ochterski, J. W.; Martin, R. L.; Morokuma, K.; Zakrzewski, V. G.; Voth, G. A.; Salvador, P.; Dannenberg, J. J.; Dapprich, S.; Daniels, A. D.; Farkas, Ö.; Foresman, J. B.; Ortiz, J. V.; Cioslowski, J.; Fox, D. J. Gaussian, Inc., Wallingford CT, 2009. 10. Boys, S. F.; Bernardi, F. Mol. Phys. 1970, 19, 553-566.

11. Parr, R. G.; Yang, W. Density functional theory of atoms and molecules; Oxford University Press: Oxford, 1989.

12. Parr, R. G.; Pearson, R. G. J. Am. Chem. Soc. 1983, 105, 7512-7516.

13. Sanderson, R. Science 1951, 114, 670-672. 